\title{
A importância da relação dos programas de pós-graduação e do setor produtivo na geração de inovação tecnológica
}

The importance of the relationship of graduate programs and the productive sector in the generation of technological innovation

La importancia de la relación de los programas de postgrado y el sector productivo en la generación de innovación tecnológica

\section{Resumo}

A disseminação e o compartilhamento dos conhecimentos produzidos pelos cursos de pós-graduação no Brasil fazem parte de uma política pública, com o propósito de estimular a inovação e consequentemente a competitividade do setor produtivo brasileiro. A presente pesquisa é um estudo de revisão de literatura, com o objetivo de compreender epistemologicamente o papel dos cursos de pós-graduação no cenário social brasileiro, traçando assim, a melhor forma de estabelecer uma gestão direcionada ao atendimento de seus fins. O estudo é de abordagem qualitativa e explicativa. $\mathrm{O}$ método adotado pela pesquisa foi a revisão narrativa, atendidos os critérios de inclusão e exclusão da amostra foram incluídos na amostra do estudo 17 estudos publicados, após 2004, na vigência da lei de inovação, que foram analisados de forma explicativa. A conclusão do estudo remonta a compreensão de que, os programas pósgraduação devem atuar estrategicamente, sempre observando as demandas de mercado e realizando suas produções de forma a atendê-las, gerando circulação de riquezas e fortalecendo produção de produtos inovadores na sociedade brasileira, inserindo o Brasil no cenário comercial internacional.

Palavras-chave: Ensino; Inovação; Pós-graduação; Gestão estratégica; Setor produtivo; Demandas.

\section{Abstract}

The dissemination and sharing of knowledge produced by postgraduate courses in Brazil are part of a public policy, with the purpose of stimulating innovation and, consequently, the competitiveness of the Brazilian productive sector. The present research is a literature review study, with the objective of epistemologically understanding the role of postgraduate courses in the Brazilian social scenario, thus outlining the best way to establish a management aimed at 
meeting its purposes. The study has a qualitative and explanatory approach. The method adopted by the research was the literature review, having met the inclusion and exclusion criteria of the sample, 17 studies published after 2004 were included in the study sample, under the validity of the innovation law, which were analyzed in an explanatory manner. The conclusion of the study goes back to the understanding that postgraduate programs must act strategically, always observing market demands and making their productions to meet them, generating circulation of wealth and strengthening the production of innovative products in Brazilian society, inserting Brazil in the international commercial scene.

Keywords: Teaching; Innovation; Postgraduate studies; Strategic management; Productive sector; Demands.

\section{Resumen}

La difusión y el intercambio de conocimientos producidos por los cursos de posgrado en Brasil son parte de una política pública, con el propósito de estimular la innovación y, en consecuencia, la competitividad del sector productivo brasileño. La presente investigación es un estudio de revisión de la literatura, con el objetivo de comprender epistemológicamente el papel de los posgrados en el escenario social brasileño, perfilando así la mejor forma de establecer una gestión orientada a cumplir con sus propósitos. El estudio tiene un enfoque cualitativo y explicativo. El método adoptado por la investigación fue la revisión narrativa, habiendo cumplido los criterios de inclusión y exclusión de la muestra, se incluyeron en la muestra de estudio 17 estudios publicados después de 2004, bajo la ley de innovación, los cuales fueron analizados de manera explicativa. La conclusión del estudio se remonta al entendimiento de que los programas de posgrado deben actuar estratégicamente, siempre observando las demandas del mercado y realizando sus producciones para atenderlas, generando circulación de riqueza y fortaleciendo la producción de productos innovadores en la sociedad brasileña, insertando a Brasil en el escena comercial internacional.

Palabras clave: Enseñando; Innovación; Posgraduación; Gestión estratégica; Sector productivo; Demandas.

\section{Introdução}

Os cursos de pós-graduação no Brasil tiveram uma estruturação definida em 1965, com a edição do Parecer Newton Sucupira e posteriormente pela Reforma Universitária que ocorreu em 1968, tendo como principal proposta a implementação de um projeto, ainda no governo militar, vinculando o ensino superior ao desenvolvimento econômico do país (Alves et al., 2014; Coelho, 2011; Cury, 2005).

Afirmam Alves et al (2014), que os programas de pós-graduação cresceram de forma exponencial nas décadas de 1970 e 1980, tornando-se assim, o aspecto mais bem sucedido da Reforma Universitária no período militar, assumindo na estrutura educacional uma posição de excelência aos demais níveis de ensino.

Estudos apontam que, o crescimento desordenado dos programas de pós-graduação exigiu a adoção de um planejamento, nascendo assim, em 1975 o PNPG (Programa Nacional de Pós-Graduação), com o objetivo de estruturar os programas de pós-graduação em um sistema de planejamento estatal, vinculado a um projeto maior do governo militar atrelando-o, mais intimamente ao desenvolvimento econômico do país (Nobre et al., 2017; Alves et al., 2014). Desta forma, o PNPG editado em 1975, vinculava os programas de pós-graduação ao PND (Plano Nacional de Desenvolvimento) (Martins, 2018).

Posteriormente, o Estado Democrático brasileiro organizado pela Constituição da República Federativa do Brasil promulgada em 1988 se preocupou em estabelecer diretrizes ao país para possibilitar o desenvolvimento tecnológico (Constituição da República Federativa do Brasil, 1988). O Congresso Nacional Brasileiro a Lei no 10.973 (2004), conhecida socialmente como a "Lei da Inovação" trazendo dispositivos legais de incentivo à inovação, à pesquisa científica e tecnológica no ambiente produtivo (Lei $\left.{ }^{\circ} 10.973,2004\right)$.

Com a edição da Lei de Inovação, o ordenamento jurídico brasileiro cria uma diretriz de incentivo a inovação, atrelando a sua importância ao desenvolvimento do país, articulando assim as universidades e os institutos de pesquisa ao setor produtivo.

O desenvolvimento de pesquisas científicas é uma atividade intimamente relacionada as instituições de ensino e aos institutos de pesquisa do país. As instituições de ensino realizam pesquisas por intermédio de seus programas de pós- 
graduação, assim tornando-se necessário compreender os papéis dos programas de pós-graduação na produção de pesquisas incrementadoras/inovadoras no país. Mais uma vez, verifica-se o vínculo dos programas de pós-graduação à política de desenvolvimento do país, sendo incompreensível o sentimento de antagonismo.

Neste cenário, o objetivo deste estudo de revisão e de abordagem qualitativa- explicativa é compreender o papel dos cursos de pós-graduação no cenário social brasileiro, traçando a melhor forma de estabelecer uma gestão direcionada ao atendimento de seus fins. O recorte temporal definido pelo estudo foram produções literárias e indicadores públicos, relacionados ao objeto deste estudo, divulgados após a vigência Lei da Inovação, ou seja, após 2004 a 2020.

\section{Metodologia}

O estudo é qualitativo e a revisão que subsidiou o marco literário do estudo foi constituída por trabalhos científicos que abordam direta ou indiretamente o objeto proposto neste artigo. A adoção do estudo qualitativo permitiu compreender subjetivamente o fenômeno social desencadeado a partir das políticas de inovação editadas pelo ordenamento jurídico brasileiro, utilizando-se da revisão literária e da análise de documentos (Lüdke et al 2015). Os referenciais bibliográficos incluídos na amostra de revisão foram selecionados por pesquisa orientada pelos seus descritores: ensino, inovação, pósgraduação, gestão estratégica, setor produtivo e demandas, sendo inicialmente selecionados 79 estudos pelo título apresentado e criteriosamente selecionado, Figura 1.

Como pode ser observado na Figura 1, a estratégia de busca realizada nas bases de dados EBSCO, Portal Capes, SCIELO, Google Academic e outras fontes identificou 79 estudos potencialmente relevantes. Destes 79 estudos, 46 foram excluídos por duplicidade, 5 foram excluídos após a leitura de títulos e resumos. Além disso, foram excluídos 11 artigos, com a leitura de textos na íntegra, pois não apresentaram relação com o estudo proposto, nem com as características estudadas. 
Figura 1 - Demonstração do Critério de busca e seleção da literatura.
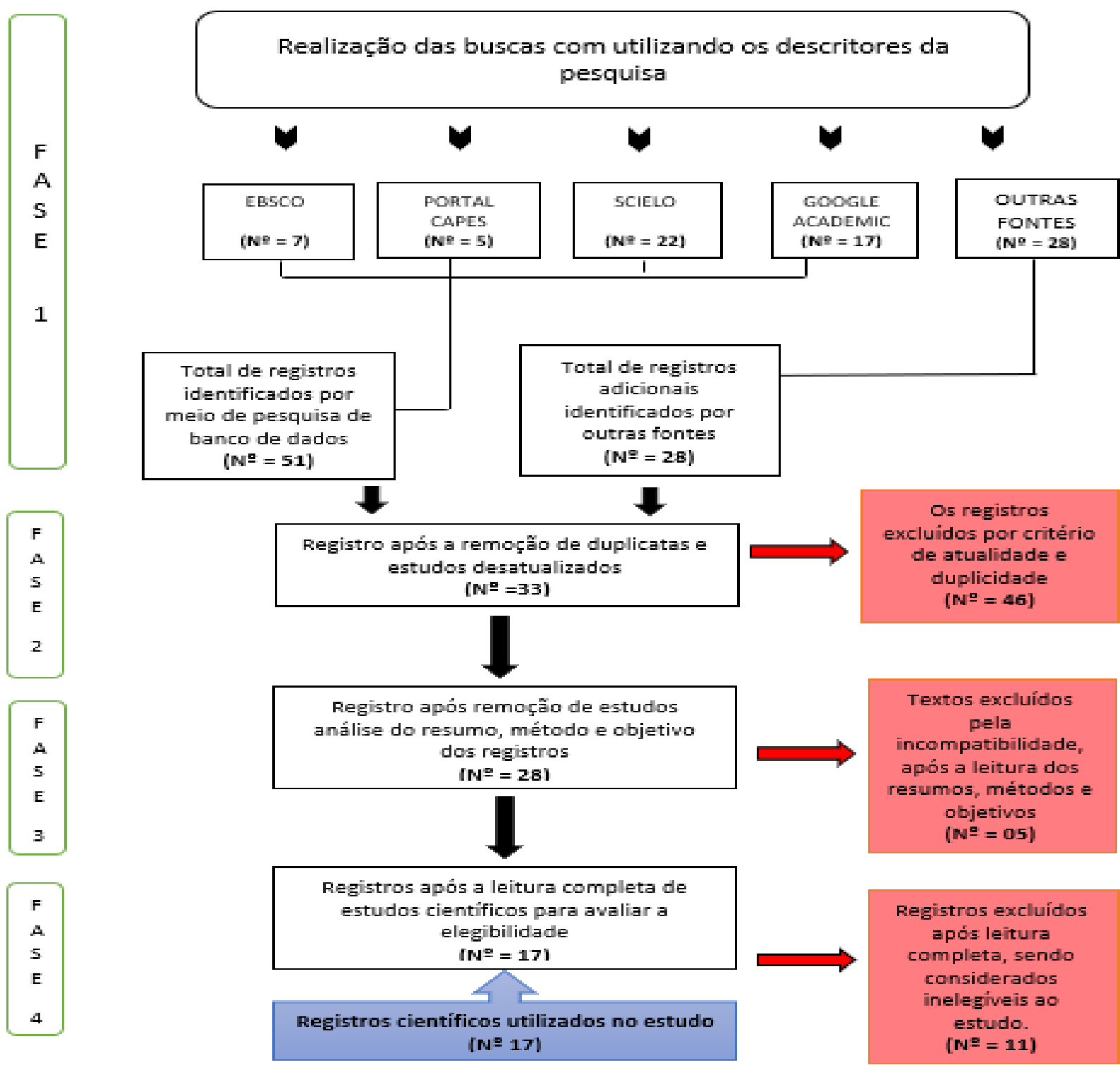

Fonte: Autores.

O critério de busca e seleção da literatura possibilitou incluir na presente pesquisa 17 estudos elaborados na vigência da lei da inovação e foram incluídos no marco literário que subsidiou o presente.

\section{Resultados e Discussão}

A produção de conhecimento no Brasil foi diretamente influenciada pelas políticas de pós-graduação, tendo como eixo a preocupação com o processo produtivo globalizado. As políticas de pós-graduação de ciência, tecnologia e inovação traçam uma orientação às universidades, com diretrizes claras à sua natureza e finalidade, bem como o direcionamento na produção de trabalhos acadêmicos em geral (Oliveira, 2013).

Martins et al. (2008), em estudo observacional, concentrou esforços para avaliar iniciativas de fortalecimento na formação de recursos humanos, na pós-graduação, para atuar no meio empresarial, estreitando a interação entre as organizações empresariais e a pós-graduação, incrementando a sua participação no processo de inovação do país. 
Afirmam, Martins et al. (2008), em sua pesquisa, que as competências e habilidades desenvolvidas nos programas de pós-graduação, não abarcam à uma necessidade do mercado, os mestres e doutores, são exímios produtores de conhecimento científico inovador, no entanto, não agregam conhecimentos e habilidades para atuar no processo produtivo empresarial. Vejamos:

Todos concordam que os profissionais oriundos da pós-graduação estão sendo formados com excelente base científica, possuem conhecimentos científicos atualizados, conhecem bem sua área de atuação, condições que os orientam a seguirem carreiras acadêmicas, isto é, estão "academicamente prontos". Entretanto, na visão dos empresários, esses profissionais não possuem habilidades em gestão, noções básicas de administração, conhecimentos de propriedade intelectual e proteção do conhecimento, construção de parcerias, entre outros aspectos fundamentais para as empresas. A frase mais repetida entre os entrevistados foi: "falta muita coisa para este profissional atuar adequadamente na iniciativa privada". (Martins et al., 2008: p. 337)

Diante desse cenário, a inserção dos profissionais atuantes nas organizações empresariais, conhecedores dos processos produtivos e das suas demandas, em programas de pós-graduação de natureza profissional, nasceu de uma estratégia do Estado brasileiro, para efetivar e potencializar a integração das universidades e das empresas na produção de inovação com aplicação industrial; e sobretudo, gerando benefícios ao desenvolvimento social e econômico brasileiro.

No mesmo sentido, se manifestam Moreira et al., (2008, p. 642):

A comunidade científica deve conceber novas políticas de formação de recursos humanos considerando que as atividades de pesquisa científica e tecnológica devem romper as barreiras entre as disciplinas acadêmicas tradicionais, pesquisa básica e aplicada, o mundo da academia e o mundo empresarial [...]. Para isso, é necessário que os novos paradigmas da ciência e tecnologia sejam colocados na pauta das discussões sobre o modelo mais adequado para a pós-graduação brasileira, seja por iniciativa da Capes, tanto quanto por iniciativa dos que atuam na gestão dos cursos em todo o país.

Em um importante estudo científico (Agopyan et al., 2005), se discutiu a importância dos mestrados profissionais para o fortalecimento da colaboração necessária entre as universidades e empresas brasileiras, em especial nos programas de pósgraduação em engenharia. O estudo conclui que, a colaboração deve ser valorizada, na medida em que, é uma forma de reconhecimento mútuo de oportunidades de desenvolvimento social e econômico.

A histórica ausência de intercâmbio de conhecimento entre as universidades e o setor produtivo, faz com que o conhecimento e a informação produzida não sejam efetivamente transferidos para as empresas (Autran et al., 2008), apesar da "importância estratégica no desenvolvimento socioeconômico brasileiro, pelo seu elevado potencial gerador de emprego, trabalho e renda" (Fujino apud Autran et al., 2008: p. 2).

O mestrado profissional nasceu com o propósito de fortalecer a relação universidades e empresas, traduzindo o conhecimento industrial qualificado em conhecimento científico, destacando os valores profissionais e de inovação, não podendo ser entendido como um demérito, em relação aos mestrados acadêmicos (Agopyan et al., 2005).

Neste sentido, afirmam Agopyan et al., (2005: p. 88):

\section{$[\ldots]$}

A pós-graduação profissional tem a sua importância reconhecida internacionalmente, em alguns países industrializados, na área das engenharias, tem-se o doutorado profissional, fortalecendo elos de ligação entre a universidade e a indústria, para o desenvolvimento.

$[\ldots]$ 
Nesta esteira de compreensão "a existência dos Programas de Mestrado Profissional é resultado de uma demanda crescente e irreversível do setor produtivo e pressupõe um ambiente legitimo e consolidado de ensino, pesquisa e desenvolvimento (P\&D) e extensão" (Quelhas et al., 2005, p. 103).

Um estudo realizado na Universidade Federal da Paraíba, com grande contribuição acadêmica, teve como objeto a aproximação do setor produtivo e da universidade. Desta forma (Autran et al., 2008), estruturaram um estudo científico, experimental, com o objetivo de identificar e analisar a transferência do conhecimento produzido por pesquisadores, professores e alunos da Universidade Federal da Paraíba (UFPB), que se enquadrassem dentro das áreas temáticas de interesse do SEBRAE, para compartilhamento com os micros e pequenos empresários (Autran et al., 2008).

A pesquisa organizou a disponibilização desses estudos, por intermédio de uma biblioteca online, após a seleção de áreas de conhecimento de interesse empresarial, realizaram o levantamento das produções, digitalizaram e depositaram na biblioteca, literaturas, artigos científicos de periódicos, capítulos de livros e publicações seriadas e estruturaram uma biblioteca online denominada Biblioteca On Line SEBRAE (Autran et al., 2008).

O estudo concluiu “que a Biblioteca On Line SEBRAE configurou-se como um repositório responsável pela gestão da informação e do conhecimento para as Pequenas e Médias Empresas (PMEs) brasileiras", que dificilmente teriam acesso aos conhecimentos (Autran et al., 2008). A transferência do conhecimento/tecnologia viabiliza a transformação em insumo e vantagem competitiva no mercado empresarial.

Para efetivar a política educacional dos programas de pós-graduação, o Governo Federal criou a Coordenação de Aperfeiçoamento de Pessoal de Nível Superior (CAPES) que é uma fundação para subsidiar o Ministério da Educação na formulação de políticas e no desenvolvimento de atividades de suporte à formação de profissionais de magistério para a educação básica e superior e para o desenvolvimento científico e tecnológico do País (Lei nº 8.405, 1992), entre suas funções está a consolidação, avaliação e fomento dos programas de pós-graduação (Silva et al., 2017).

Atualmente no Brasil existem 4.663 programas de pós-graduação periodicamente avaliados e reconhecidos pela CAPES, em todo o território federativo brasileiro (Capes, 2020). Nos últimos anos houve crescimento no número de programas reconhecidos e credenciados pela CAPES, (Figura 2). 
Figura 2 - Crescimento dos cursos de pós-graduação na última década - período de 2007 - 2017.

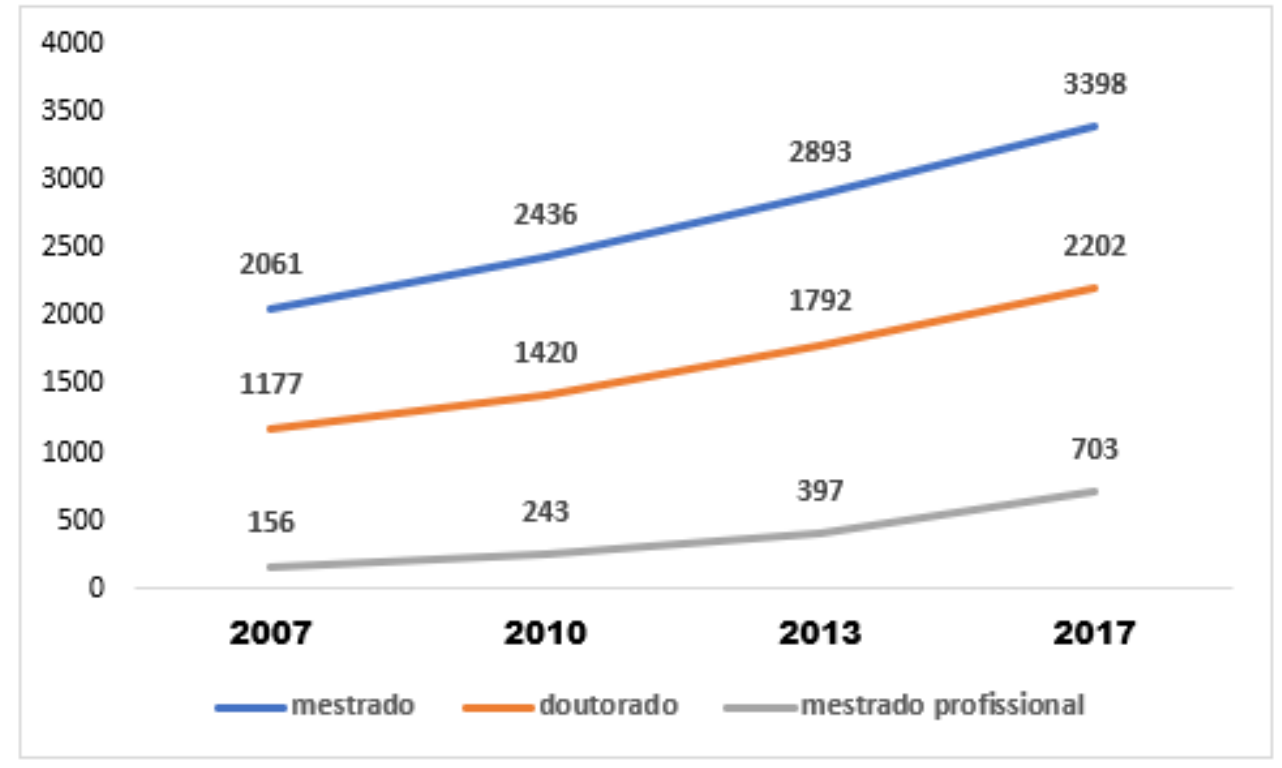

Fonte: Capes (2017).

Na avaliação do número de programas de pós-graduação no país no período compreendido entre 2007 à 2017 , os números de programas de mestrados acadêmicos cresceram 64,9\%, os números de programas de doutorados acadêmicos apresentaram crescimento de $87,1 \%$, mas o maior índice de crescimento foi dos mestrados profissionais que apresentaram um crescimento de $350,6 \%$, Figura 2.

O expressivo crescimento dos programas de pós-graduação profissional no país, no período analisado, sinaliza para a existência de uma demanda reprimida, com maior intimidade com as demandas do mercado.

Os programas de pós-graduação periodicamente são avaliados pela CAPES, sendo que os resultados da avaliação são expressos em escala de 1 a 7, os programas com notas inferiores a 3 são descredenciados, sendo que os programas de pósgraduação com notas 6 e 7 são reconhecidos como cursos com desempenho equivalente ao alto padrão internacional (Capes, 2020).

No mesmo período em que houve crescimento dos programas de pós-graduação no país, ou seja, no período de 2007 à 2017, os programas de pós-graduação apresentaram aumento em seus padrões de qualidade. Os programas com desempenho equivalente aos padrões internacionais tiveram um crescimento de 99,6\% no país, Figura 3. 
Figura 3 - Programas de Excelência classificados pela Capes/MEC - período de 2007 - 2017.

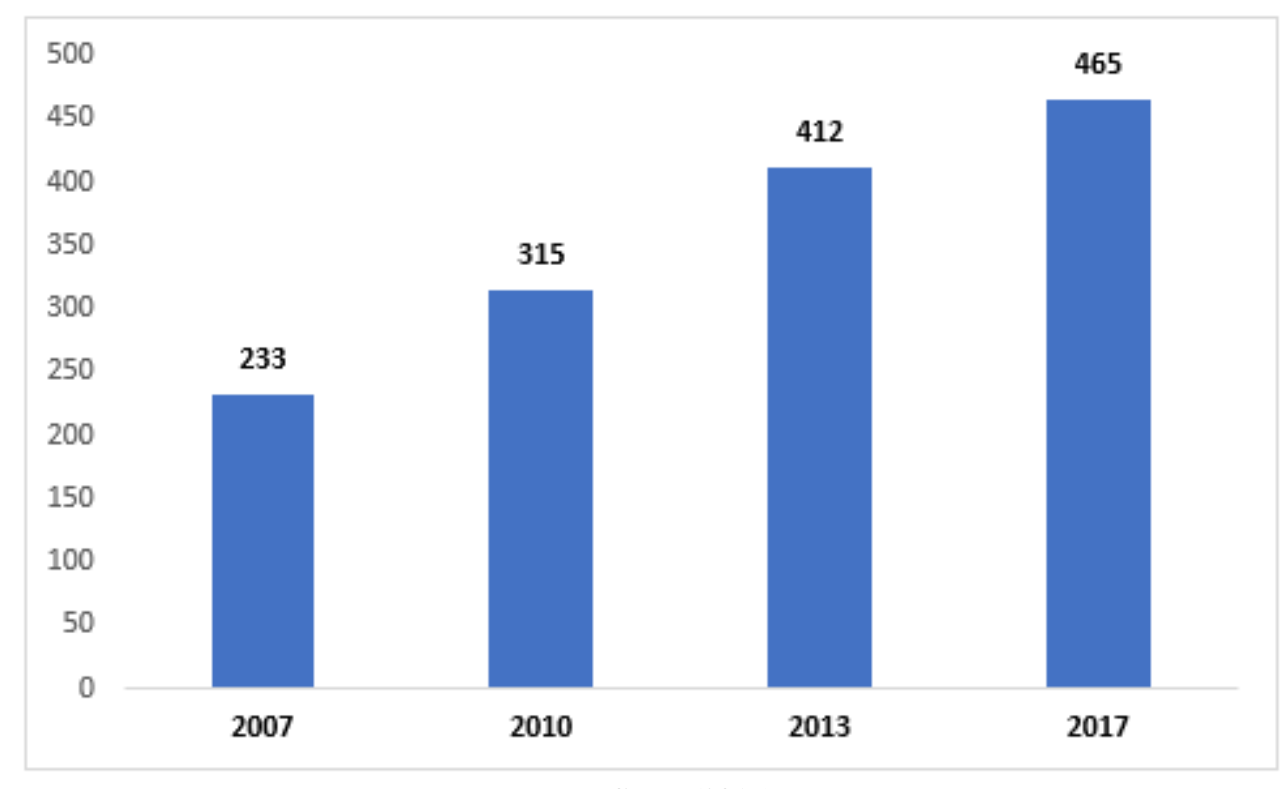

Fonte: Capes (2017).

O crescimento do número de programas em território brasileiro e o aumento em seu padrão de qualidade, coincidiu como o crescimento apontado no número de títulos de pós-graduação concedidos no período de 2010 a 2016, Figura 4.

Figura 4 - Número de alunos titulados em programas de pós-graduação - período de 2010 - 2016, distribuídos por título e ano.

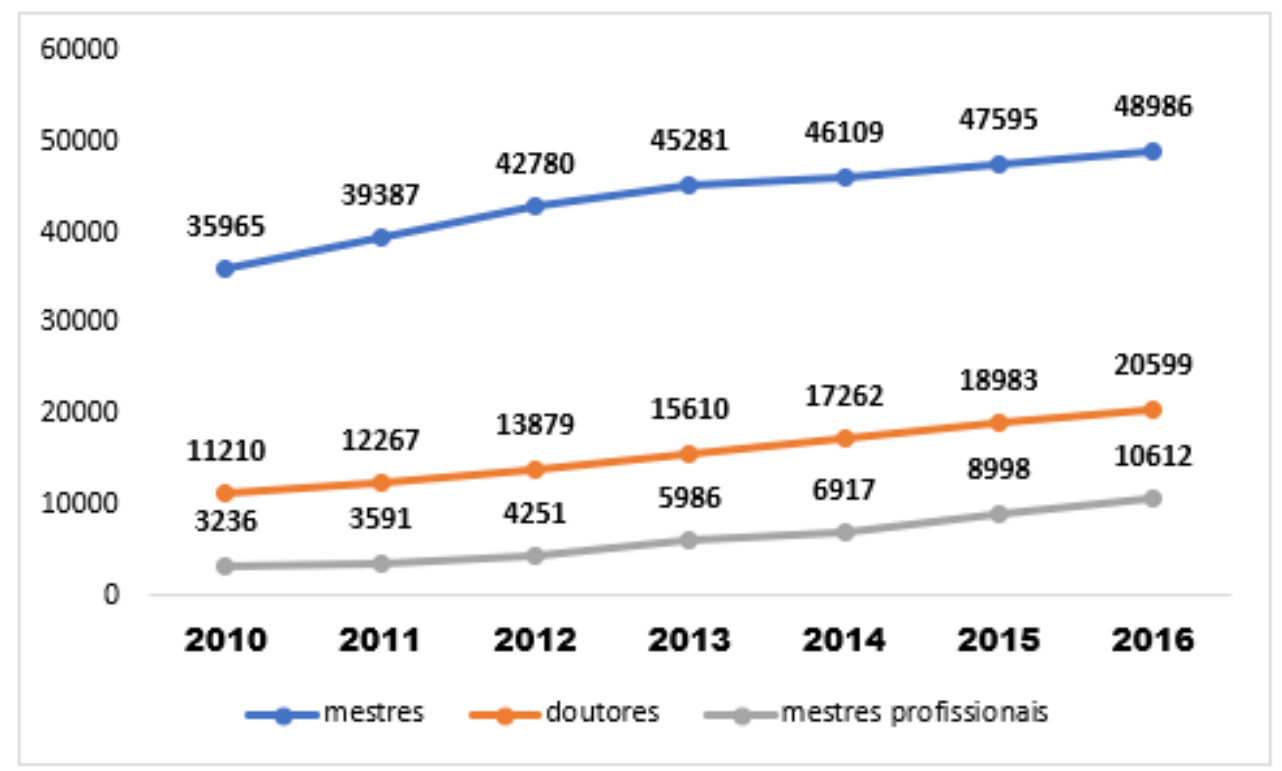

Fonte: Capes (2017).

Entre 2010 e 2016, a análise dos títulos concedidos pelos programas de pós-graduação no Brasil, aponta para um crescimento de títulos de mestres acadêmicos de 36,2\%, doutores acadêmicos de 83,7\% e mais uma vez os títulos de mestres profissionais representam um crescimento superior ao demais programas, ou seja, $227,9 \%$.

O crescimento no número de programas e o aumento no número de títulos concedidos refletiram diretamente no volume de produção científica produzida pelo Brasil, Figura 5. 
Figura 5 - Número de publicação de artigos científicos, livros e capítulo de livros - período de 2010 - 2016.

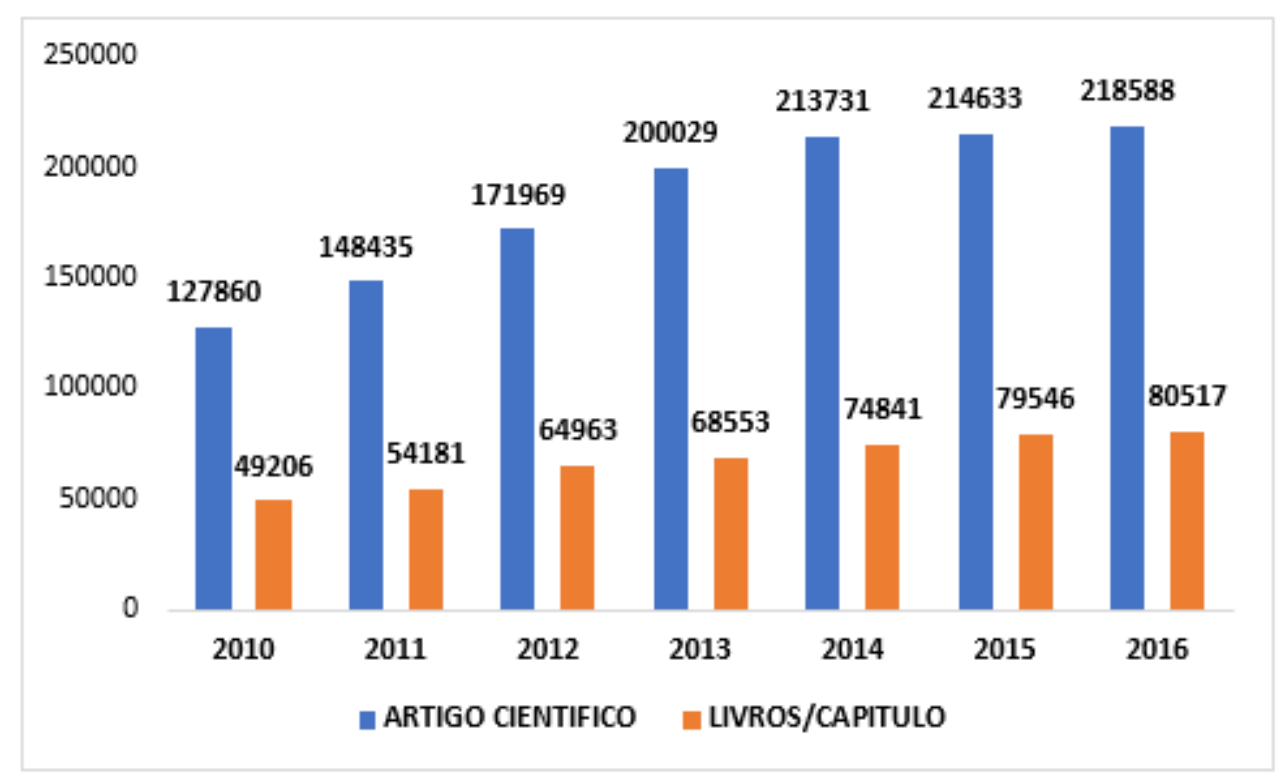

Fonte: Capes (2017).

Entre 2010 e 2016, o volume de produções científicas na forma de livros ou capítulo de livros e artigos científicos foram avaliados. Os artigos científicos tiveram um aumento de 70,9\%, sendo que a produção em livros ou capítulos de livros apresentaram um crescimento de 63,6\%, atingindo em 2016 uma produção acadêmica aproximada a 300.000 estudos científicos.

O elevado volume de produção científica demonstra a capacidade de produção de estudos inovadores capazes de gerar insumos importantíssimos para aplicação no setor produtivo do país. Entre os Estados brasileiros com maior número de produção científica, os três estados com maior número de produção são: São Paulo, Rio de Janeiro e Minas Gerais demonstrando uma maior concentração de estudos realizados na região sudeste, Figura 6.

O Brasil mantém elevado número de produções científicas incluídas na Web of Science com colaboração internacional, sendo que desses estudos muitos estão classificados entre os Top 10\% e Top 1\%, demonstrando a qualidade e a importância dos estudos brasileiros no cenário mundial, Figura 6. 
Figura 6 - Os perfis dos dez estados brasileiros com a maior produção de pesquisa científica, período 2011 - 2016.

\begin{tabular}{|c|c|c|c|c|c|c|}
\hline State & $\begin{array}{l}\text { Web of } \\
\text { Science } \\
\text { Documents }\end{array}$ & $\begin{array}{c}\text { Category } \\
\text { Normalized } \\
\text { Citation Impact }\end{array}$ & $\begin{array}{c}\% \text { Documents } \\
\text { in Top } 1 \%\end{array}$ & $\begin{array}{c}\% \text { Documents } \\
\text { in Top } 10 \%\end{array}$ & $\begin{array}{c}\% \text { Industry } \\
\text { Collaborations }\end{array}$ & $\begin{array}{c}\% \\
\text { International } \\
\text { Collaborations }\end{array}$ \\
\hline Rio De Janeiro & 39,996 & 0.93 & 1.15 & 7.80 & 2.28 & 37.89 \\
\hline Minas Gerais & 36,660 & 0.76 & 0.64 & 5.61 & 0.79 & 27.33 \\
\hline Santa Catarina & 12,312 & 0.84 & 0.63 & 6.08 & 0.89 & 29.11 \\
\hline Pernambuco & 10,589 & 0.71 & 0.54 & 5.18 & 0.85 & 26.92 \\
\hline Distrito Federal & 10,584 & 0.94 & 1.22 & 6.68 & 0.94 & 36.27 \\
\hline Bahia & 9,189 & 0.73 & 0.78 & 5.27 & 0.79 & 28.11 \\
\hline Ceara & 7,559 & 0.76 & 0.73 & 5.97 & 0.74 & 28.58 \\
\hline Para & 5,148 & 0.81 & 0.99 & 5.94 & 0.70 & 32.69 \\
\hline Espirito Santo & 3,837 & 0.63 & 0.60 & 4.56 & 1.33 & 24.68 \\
\hline Amazonas & 3,735 & 0.81 & 1.12 & 6.93 & 0.64 & 34.40 \\
\hline Mato Grosso Do Sul & 3,541 & 0.56 & 0.34 & 3.33 & 0.37 & 16.83 \\
\hline Mato Grosso & 3,209 & 0.62 & 0.87 & 3.99 & 0.28 & 19.82 \\
\hline Sergipe & 2,658 & 0.72 & 0.56 & 5.38 & 0.64 & 24.23 \\
\hline Piaui & 2,066 & 0.53 & 0.15 & 3.87 & 0.19 & 16.46 \\
\hline Alagoas & 1,819 & 0.71 & 0.38 & 6.05 & 0.44 & 29.41 \\
\hline Maranhao & 1,715 & 0.73 & 0.52 & 4.14 & 0.35 & 20.00 \\
\hline Tocantins & 900 & 0.49 & 0.22 & 2.56 & 0.44 & 19.44 \\
\hline
\end{tabular}

Fonte: Research in Brazil (2017).

A análise das publicações de estudos científicos na Web of Science ${ }^{1}$, no período de 2011 a 2016, um dos principais veículos de divulgações de estudos científicos internacionais de alto impacto, revela que o Brasil atingiu a $13^{\mathrm{a}}$ colocação, entre os maiores produtores de pesquisas científicas de alta qualidade, no mundo.

O estudo chama atenção para a problemática levantada pelo presente estudo, ou seja, em todos os estados, nenhum deles apresentam altos índices de colaboração das indústrias brasileiras na realização da pesquisa. Deste modo, o Estado brasileiro que apresentou maior colaboração industrial na pesquisa, no período analisado, foi o Rio de Janeiro com 2,3\% das pesquisas, Figura 6.

A liderança em número de estudos científicos é dos EUA, que despontam com um volume de $80 \%$ a mais de produção científica do que a China, segunda colocada no ranking mundial, Figura 7.

\footnotetext{
${ }^{1}$ A Web of Science é uma plataforma referencial de citações científicas projetada para apoiar pesquisas científicas e acadêmicas com cobertura nas áreas de ciências, ciências sociais, artes e humanidades. O Web of Science Core Collection é o principal recurso na plataforma e inclui mais de 20.000 revistas acadêmicas de alta qualidade revisadas por pares e publicadas em todo o mundo (incluindo periódicos de Acesso Aberto), e mais de 190.000 processos de conferências.
} 
Figura 7 - Artigos científicos adicionados ao Web of Science, período de 2011 - 2016.

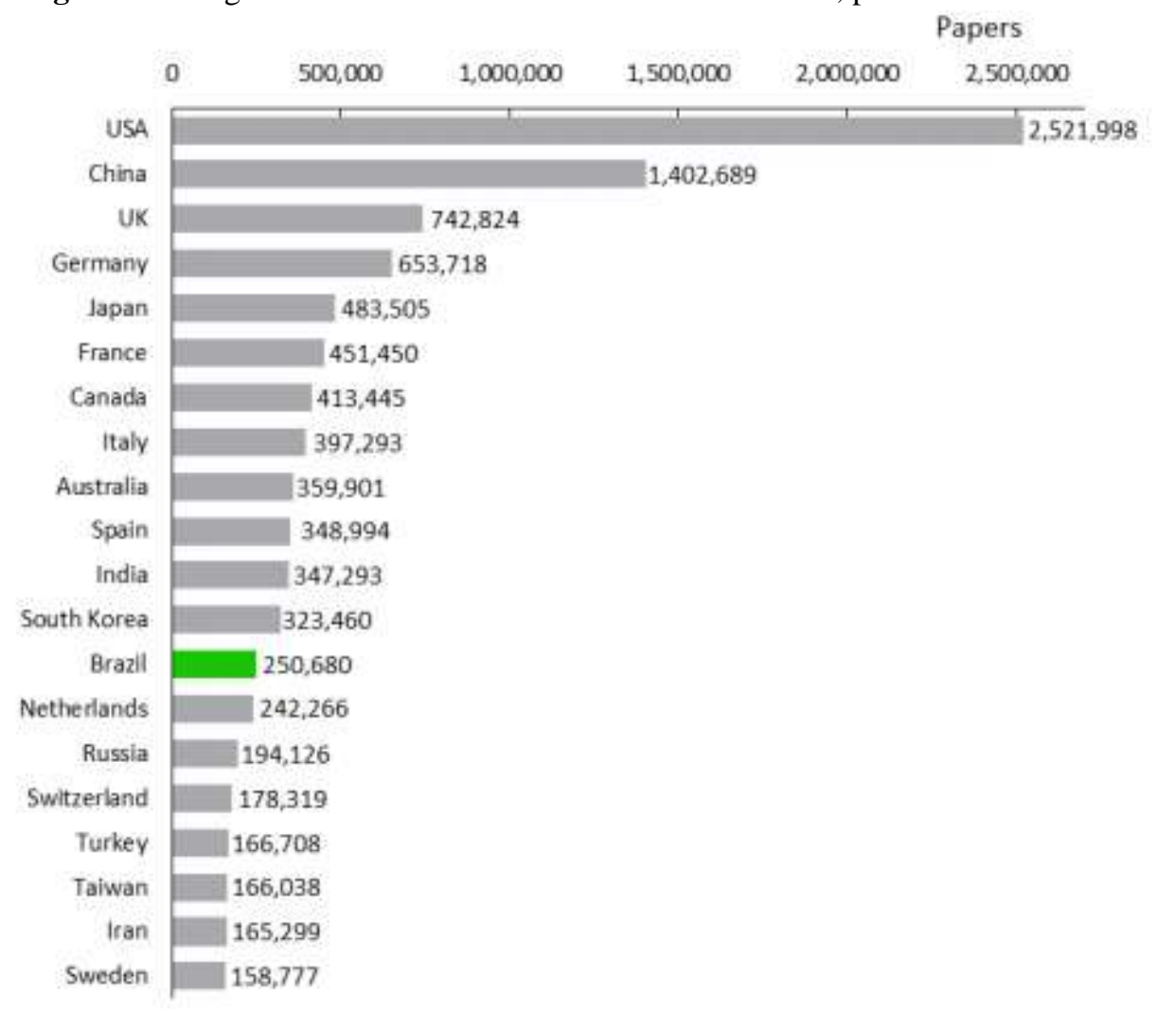

Fonte: Research in Brazil (2017).

A Universidade de São Paulo (USP), a Universidade de Campinas (UNICAMP) e a Universidade Estadual Paulista (UNESP) são grandes produtoras de estudos científicos de alta qualidade, sendo ranqueadas internacionalmente, entre as melhores do mundo, assim afirma Marcovitch et al., 2018: p. 73:

Uma análise mais apurada da edição de 2017 do arwu-gras ${ }^{2}$ mostra que as melhores posições de universidades brasileiras foram a da Unicamp (6 ${ }^{\mathrm{a}}$ posição) e a da usp ( $7^{\mathrm{a}}$ posição), classificadas entre as Top 10 do mundo em Ciência e Tecnologia de Alimentos. Além desse destacado desempenho, a USP ficou entre as cinquenta melhores do mundo em mais três áreas: $9^{a}$ em Odontologia, $12^{a}$ posição em Agricultura, 39 em Biotecnologia. A usp foi seguida pela Unesp, que alcançou a $40^{a}$ posição em Agricultura e em Odontologia e a $41^{\mathrm{a}}$ em Veterinária. A Unicamp, além da posição de destaque em Ciência e Tecnologia de Alimentos, ocupou também em $44^{\mathrm{a}}$ posição em Odontologia.

O Brasil reconhecidamente no cenário mundial é um grande produtor de pesquisa científica de qualidade, com universidades de reconhecimento internacional.

\section{Considerações Finais}

No Brasil, a vida acadêmica sempre foi alimentada pelo sentimento de antagonismo com a vida no setor produtivo. Tal sentimento criou ao longo da história um distanciamento, tornando a academia um ambiente de produção acadêmica

\footnotetext{
${ }^{2}$ Academic Ranking of World Universities - Global Ranking of Academic Subjects (ARWU-GRAS)
} 
divorciada das demandas de mercado, ocupou-se com produções científica e conhecimentos meramente filosóficos, oferecendo resistência em oferecer contribuições aos setores produtivos e as demandas de mercado no Brasil.

O estudo de revisão possibilitou concluir que desde sua origem, os programas de pós-graduação estiveram intimamente relacionados as estratégias e políticas governamentais, com o objetivo de efetivar o desenvolvimento do país, por intermédio de sua articulação com o setor produtivo. Sendo certo que, as produções científicas brasileiras são de altíssima qualidade, mundialmente reconhecida.

Compreender a sociedade, suas necessidades e demandas é o objeto de ocupação dos programas de pós-graduação, que deve utilizar a sua capacidade de produção científica incrementadora/inovadora ao atendimento das necessidades sociais, em articulação com o setor produtivo, melhorando a qualidade de vida dos cidadãos. Somente assim, os programas atenderão o seu fim, que é contribuir com o desenvolvimento econômico e social do país.

O desenvolvimento de pesquisas com aplicabilidade empresarial, potencializa, invariavelmente, a possibilidade de fomentos empresariais para as pesquisas; e ainda, o investimento do setor privado vislumbrará nos programas de pósgraduação um terreno fértil para o fortalecimento de sua competitividade, em um mercado globalizado.

Nesse sentido, o desenvolvimento de pesquisas, desde seus projetos estruturais, necessita abarcar justificativa e demanda mercadológica, mediata ou imediata, gerando circulação de riquezas e produtos inovadores no mercado de consumo, não devendo se limitar a produções de pesquisas meramente acadêmicas e sem qualquer aplicabilidade, distanciando-se da sociedade e do seu propósito constitucional, o que viabilizará maior captação de recursos financeiros para desenvolvimento das pesquisas.

A conclusão deste estudo abre espaço para o desenvolvimento de outros estudos, com maior detalhamento sobre as contribuições dos cursos de pós-graduação no acesso a fomentos e uma melhor análise do perfil e das áreas de concentração das pesquisas de impacto realizadas, nos últimos anos, pelas universidades brasileiras.

\section{Referências}

Agopyan, Y., \& Oliveira, J. F. G. (2005). Mestrado profissional em Engenharia: uma oportunidade para incrementar a inovação colaborativa entre universidades e os setores de produção no Brasil. R B P G, 2 (4). http://ojs.rbpg.capes.gov.br/index.php/rbpg/article/view/80/77

Alves, M. F., Ferreira, J., \& Oliveira, D. E. (2014)). Pós-Graduação no Brasil: do Regime Militar aos dias atuais Undergraduate in Brazil: the Military Regime to the present day Posgrado en Brasil: de el Régimen Militar hasta nuestros días, 30 (Issue 2). https://doi.org/10.21573/vol30n22014.53680

Autran M. M. M., Arruda Ramalho F., Dantas Benício, C., Macedo, C., \& Dantas Ferreira, E. (2008). A transferência do conhecimento para o setor produtivo: experiência de uma parceria (Issue 2). https://brapci.inf.br/_repositorio/2010/11/pdf_3eebb94b46_0013273.pdf

Capes (2017). Coordenação de Aperfeiçoamento de Pessoal de Nível Superior. Resultado da $1^{a}$ etapa avaliação quadrienal. https://www.gov.br/capessala-deimprensa/noticias/8557-divulgado-o-resultado-da-1-etapa-da-avaliacao-quadrienal-2017.

Capes. (2020). Coordenação de Aperfeiçoamento de Pessoal de Nível Superior. https://sucupira.capes.gov.br/sucupira/public/consultas/ coleta/programa/quantitativos/quantitativoPrograma.jsf?areaAvaliacao=48\&areaConhecimento

Coelho M., \& Hayashi, M. C. (2011). Pós-graduação no regime militar: zona franca de produção do conhecimento In Série-Estudos: Periódico do Programa de Pós-Graduação em Educação da UCDB, Campo Grande - MS (31), 193-213. https://serie-estudos.ucdb.br/serie-estudos/article/view/135

Constituição da República Federativa do Brasil. (1988). http://www.planalto.gov.br/ccivil_03/Constituicao/Constituicao.htm

Cury, C. R. J. (2005). Quadragésimo ano do parecer CFE no 977/65. Revista Brasileira de Educação, 1931(30), 07-20. https://doi.org/10.1590/s141324782005000300002

Kneipp J. M., Rosa L. A. B, Bichueti R. S., Perlin A. P., \& Schuch Junior V. F. (2011). Uma análise da evolução da produção científica sobre inovação no brasil. Rev. eletr. estrat. neg., 4 (1), 133-157. http://portaldeperiodicos.unisul.br/index.php/EeN/article/view/597

Lei $n^{\circ}$ 8.405, de 9 de janeiro de 1992. (1992). Autoriza o Poder Executivo a instituir como fundação pública a Coordenação de Aperfeiçoamento de Pessoal de Nível Superior (Capes) e dá outras providências. http://www.planalto.gov.br/ccivil_03/LEIS/L8405.htm

Lei $n^{o} .10 .973$, de 2 de dezembro de 2004 (2004). Dispõe sobre incentivos à inovação e à pesquisa científica e tecnológica no ambiente produtivo e dá outras providências. http://www.planalto.gov.br/ccivil_03/_Ato2004-2006/2004/Lei/L10.973.htm

Lüdke, M, \& André, M. E. D. A. (2015). Pesquisa em educação: abordagens qualitativas. EPU. 
Research, Society and Development, v. 10, n. 4, e51010414342, 2021

(CC BY 4.0) | ISSN 2525-3409 | DOI: http://dx.doi.org/10.33448/rsd-v10i4.14342

Marcovitch, J., Goldemberg, J., Axel-Berg, J., Righetti, S., Santos, S. M. dos, Oliveira, L. N. de, Grácio, M. C. C., Rosas, F. S., Ranieri, N., Pedrosa, R., Pereira, M. W., Cruz, C. H. de B., Shimizu, K., Ferreira, J. E., Machado, R., Segurado, A. C., Beppu, M. M., Holland, H., \& Guimarães, J. A. C. (2018) Repensar a universidade: desempenho acadêmico e comparações internacionais. In Repensar a universidade: desempenho acadêmico e comparações internacionais. Universidade de São Paulo. Escola de Comunicações e Artes. https://doi.org/10.11606/9788571661868

Martins, C. B. (2018). As origens pós-graduação nacional (1960-1980). Revista Brasileira de Sociologia - RBS, 6(13). https://doi.org/10.20336/rbs.256

Martins, C. B., Lúcia, A., \& Assad, D. (2008). A pós-graduação e a formação de recursos humanos para inovação. RBPG, Brasília, 5 (10), $322-352$. http://ojs.rbpg.capes.gov.br/index.php/rbpg/article/view/157/151

Moreira M. L., \& Velho L. (2008). Pós-graduação no brasil: da concepção "ofertista linear" para "novos modos de produção do conhecimento" implicações para avaliação. Avaliação, 13 (3), 625-645. https://doi.org/10.1590/S1414-40772008000300002

Nobre L. N., \& Freitas R. R. (2017). A Evolução da Pós-Graduação no Brasil: Histórico, Políticas e Avaliação. Brazilian Journal of Production Engineering, São Mateus, 3, (2), 18-30. https://doi.org/10.0001/v3n2_3

Oliveira, J. (2013) A política de ciência, tecnologia e inovação, a pós-graduação e a produção do conhecimento no Brasil. Inter-Ação, Goiânia, 38 (2), 323 338. https://repositorio.bc.ufg.br/bitstream/ri/13857/5/Artigo\%20-\%20Jo\%C3\%A3o\%20Ferreira\%20de\%20Oliveira\%20-\%202013.pdf

Quelhas O. L. G., Faria Filho J. R., \& França, S. L. B. (2005). O mestrado profissional no contexto do sistema de pós-graduação brasileiro. RBP G, 2 (4) 97104. https://doi.org/10.21713/2358-2332.2005.v2.82

Research in Brazil (2017). A report for CAPES by Clarivate Analytics. Clarivate Analytics. http://portal.andes.org.br/imprensa/noticias/imp-ult-992337666.pdf

Silva I. C., Farias L. A., \& Santos W. (2017). Pós-graduação e produção científica: a teoria do capital humano e as demandas do setor produtivo. Filosofia e Educação [RFE]- 8 (3) $142-168$,

Silva Junior, J. D., \& Sguirssadi, V. (2013). Universidade Pública Brasileira no Século XXI Educação superior orientada para o mercado e intensificação do trabalho docente. Espac. blanco, Serie. indagaciones, 23 (1), 119 - 156. https://www.redalyc.org/pdf/3845/384539805007.pdf 